\title{
Carrier Tracking using Extended Kalman Filters for GNSS Synthetic Aperture Processing with a Rotating Antenna
}

\author{
Miguel Angel Ribot, École Polytechnique Fédérale de Lausanne (EPFL), Switzerland \\ Joaquín Cabeza, École Polytechnique Fédérale de Lausanne (EPFL), Switzerland \\ Pau Closas, Electrical and Computer Engineering Dept., Northeastern University, Boston, MA, USA \\ Cyril Botteron, École Polytechnique Fédérale de Lausanne (EPFL), Switzerland \\ Ferran Valdés, Universitat Illes Balears, Spain \\ Bartomeu Alorda, Universitat Illes Balears, Spain \\ Pierre-André Farine, École Polytechnique Fédérale de Lausanne (EPFL), Switzerland
}

\section{BIOGRAPHY}

Mr. Miguel Angel Ribot received a BSc degree in Telematics Engineering from the Universitat de les Illes Balears (UIB) in 2008, and the MSc in Information and Communication Technologies from the Polytechnic University of Catalonia (UPC) in 2011. He is currently working towards the PhD degree in GNSS signal processing and GNSS-Reflectrometry in the Electronics and Signal Processing Laboratory at the École Polytechnique Fédérale de Lausanne (EPFL). His PhD has been supported by the Swiss National Science Foundation (SNSF).

Mr. Joaquín Cabeza received his BSc and MSc in Telecommunications Engineering from Universidad Politécnica de Madrid (UPM) in 2014 and 2016 respectively. In February 2016 he joined the Electronics and Signal Processing Laboratory (ESPLAB) at the École Polytechnique Fédérale de Lausanne (EPFL), Switzerland where he is currently collaborating as a Scientific Assistant. Among others, his research interests include GNSS signal processing, antenna arrays and synthetic aperture antennas.

Dr. Pau Closas is Assistant Professor at Northeastern University, Boston, MA. He received the MSc and PhD degrees in Electrical Engineering from UPC in 2003 and 2009, respectively. He also holds a MSc degree in Advanced Mathematics and Mathematical Engineering from UPC since 2014. His primary areas of interest include statistical signal processing, robust stochastic filtering, and game theory, with applications to positioning systems and wireless communications. He is the recipient of the 2014 EURASIP Best PhD Thesis Award, the 9th Duran Farell Award, and the 2016 ION Early Achievements Award.

Dr. Cyril Botteron is leading, managing, and coaching the research and project activities of the GNSS and Ultra-Wideband and mm-wave groups at École Polytechnique Fédérale de Lausane (EPFL). He is the author or co-author of 5 patents and over 110 publications in major journals and conferences in the fields of wireless positioning systems, GNSS-based navigation 
and sensing, ultra-low-power radio frequency communications and integrated circuits design, and baseband analog and digital signal processing.

Mr. Ferran Valdés received his BSc degree in industrial engineering from the Universitat de les Illes Balears (UIB) in 2013. He is currently working on his MSc in Communications Engineering at the Rheinisch-Westflische Technische Hochschule Aachen (RWTH). His research interests include wireless communications, radar and embedded systems.

Dr. Bartomeu Alorda received the B.S. degree from the University of Balearic Islands, in 1995, and the M.S. in Telecomunication Engineering from Polytechnic University of Catalonia in 2000. The PhD degree in Physics was received from the University of Balearic Islands in 2005. He is currently an associated professor at the Illes Balears University (UIB). The main interests are related to microelectronic reliability issues and the exploration of new wireless devices applications to improve their capabilities in the IoT context.

Prof. Pierre-André Farine is professor in electronics and signal processing at EPFL, and is head of the electronics and signal processing laboratory. He received the MSc and PhD degrees in Micro technology from the University of Neuchtel, Switzerland, in 1978 and 1984, respectively. He is active in the study and implementation of low-power solutions for applications covering wireless telecommunications, ultra-wideband, global navigation satellite systems, and video and audio processing. He is the author or co-author of more than 120 publications in conference and technical journals and 50 patent families (more than 270 patents).

\section{ABSTRACT}

A single GNSS antenna moving along a known trajectory can be used to synthesize a virtual array in order to apply spatial diversity techniques, e.g. beamforming. With these techniques, referred as synthetic aperture (SA) techniques, the receiver can mitigate interfering signals, including multipath. The use of a single antenna element, instead of an antenna array, significantly reduces the hardware complexity, and there is no longer need for precise calibration and system synchronization. Before SA techniques can be used to process the GNSS signal, a critical practical issue must be addressed: the carrier Doppler frequency caused by the antenna motion only, that we have called "relative" Doppler, must be isolated from any other carrier frequency contribution. We have called the sum of all these possible contributions "reference" Doppler. In this paper, we propose two new techniques making use of the so-called extended Kalman filter (EKF), in order to compensate the reference Doppler at the correlation output. The first method, named EKF1, tracks the carrier frequency using a conventional FLL, and then uses its output to feed an EKF responsible for the reference Doppler estimation. The second method, named EKF2, is an ultra-tight integration solution in charge of the carrier tracking, while simultaneously estimating the reference Doppler component from the correlators output. A comparison of these new methods with two previously existing techniques, in terms of their impact on direction-of-arrival estimation techniques, is presented. Synthetic and real GPS L1 C/A signals are used in this comparison. Real signal measurements were obtained using a GPS antenna mounted on a mechanical rotating arm -built in-house- to implement an approximately uniform circular movement.

\section{INTRODUCTION}

Global Navigation Satellite Systems (GNSS) receivers have become ubiquitous in modern society. Many of these GNSS receivers are expected to operate in dense urban environments where severe multipath presence is expected [1]. Multipath is the main source of natural interference degrading the accuracy and reliability of the GNSS measurements. Multipath effects are particularly hard to mitigate since they depend on each specific reception scenario. During the last two decades, the GNSS community has developed several techniques to increase the receivers robustness against multipath [2]. Among these techniques, spatial filtering using antenna arrays, also referred to as beamforming, has been found to be particularly effective. Since it is very likely that the GNSS line-of-sight (LOS) signal and the multipath will have different direction-of-arrival (DOA), the spatial diversity provided by the antenna arrays can be used to amplify signals coming from certain directions and attenuate signals coming from others. This is known as beamforming. Moreover, this same diversity allows to also estimate the DOA of the received signals. Unfortunately, the size of the GNSS antenna arrays is a big limitation for their usage in many applications. Besides, using beamforming techniques increases the receivers hardware complexity and, in many cases, the required computing 
power. As an alternative to traditional antenna arrays, synthetic aperture (SA) techniques also allow spatial filtering using a single antenna element in motion. SA has been used for decades in the remote sensing field [3]. In the GNSS context, the use of SA techniques was proposed in [4-8], mostly for indoors and dense multipath environments. In [7], the authors adapt some well-known beamforming algorithms, such as the minimum power distortionless response (MPDR), and DOA algorithms, such as MUSIC, for its use with synthetic aperture at post-correlation level, given a known uniform circular antenna trajectory.

The existing SA GNSS research identifies an important practical challenge that must be addressed before applying any SA technique: the carrier frequency observed by the moving antenna has some unwanted contributions that must be compensated. Without considering the noise contribution, SA techniques require that any observed carrier phase variation has to be caused by the relative movement of the antenna with the origin of coordinates of a defined spatial reference frame. We call this carrier frequency shift relative Doppler. The origin of coordinates can be arbitrarily selected, e.g. the starting point of the trajectory, or its center. In addition to the relative Doppler, the observed carrier frequency also includes the contributions of the relative movement of the satellite with the origin of coordinates, the shift due to the carrier phase wrap-up [9], errors due to effects of the propagation across the atmosphere, and clock errors [10]. We call reference Doppler the sum of all these contributions.

Even in an interference free scenario where only LOS signal is received, the isolation of the relative Doppler shift is not straightforward. In [7], the authors describe the following two methods in order to do so. A first method, consists in approximating the reference Doppler as linear and use a least squares estimation (LSE) algorithm to estimate the regression coefficients, which correspond to a constant frequency shift $f_{d}$ and a frequency rate $\dot{f}_{d}$. The input to this LSE algorithm is a batch of carrier frequency estimates obtained from the carrier tracking loop. The length of the selected batch strongly impacts the LSE accuracy. In this paper, we call this method linear interpolation method. A second method consists in using an additional static antenna, ideally placed in the origin of coordinates of the considered reference frame, to track the reference Doppler. The carrier frequency observed by the static antenna is dominated by the relative satellite-receiver motion. Hence, the reference Doppler estimate obtained with the static antenna can be used to compensate the total carrier frequency observed by the moving antenna, thus isolating the relative Doppler. However, this approach increases the receiver complexity and the amount of required signal processing. Moreover, having an extra static antenna can be unfeasible for some applications. We call this method reference static antenna method.

In this paper, we propose a new approach using an extended Kalman filter (EKF) to effectively decouple the Doppler contributions in order to overcome the limitations of the existing methods. The EKF sequentially updates the signal parameter estimates, which naturally solves the issue of the LSEs batch length selection faced in the linear interpolation method, and does not require any extra static antenna element.

When a state-model describing the tracked carrier phase is available, Kalman filtering techniques are preferred as they are an efficient approach to estimate time-varying parameters. Particularly, in the EKF, the nonlinear state transition and observation models -as long as they are differentiable- are linearized about the working point [11]. The extra phase component due to the relative Doppler can be included within the state-model. If the parameter estimation is successful, both Doppler contributions can be separated and spatial filtering techniques can be used. More specifically, in this paper we propose two different EKF implementations, which we will refer as EKF1 and EKF2. In EKF1, the receiver tracks the carrier frequency using a traditional frequency locked-loop (FLL). The carrier frequency estimate is fed to the EKF which tries to estimate the reference Doppler contribution. This estimate is used to correct the output of the correlators. As a result, the only remaining carrier frequency is due to relative Doppler contribution. In EKF2, we implement an ultra-tightly integration architecture [11], in which an EKF is in charge of the carrier tracking while simultaneously removing the reference Doppler component from the correlators output.

\section{SIGNAL MODEL AND SCENARIO DEFINITIONS}

After compensating for the nominal carrier frequency $f_{c}$, the complex baseband representation of the received GNSS signal at the antenna output at time instant $t$ can be expressed as $[2,10]$

$$
x(t)=\sum_{l=1}^{L} a_{l}(t) s_{l}\left(t-\tau_{l}(t)\right) \exp \left\{-j 2 \pi f_{c} \tau_{l}(t)\right\}+n(t)
$$


where $L$ is the number of satellites in view. $s_{l}(t)$ is the GNSS navigation signal spread by the pseudorandom code of the $l$ th satellite. $a_{l}(t), \tau_{l}(t)$ are the complex amplitude and the time delay of the $l$ th satellite signal, respectively. $n(t)$ represents additive noise and any other possible disturbing terms, such as interferences or multipath. Without loss of generality, we will assume a single satellite in view in our analysis. This assumption is reasonable given the low cross-correlation among the pseudorandom codes from different satellites [10]. Therefore, from now on we drop the subscript $l$ in order to simplify our notation.

For a moving receiver, we can approximate the delay $\tau(t)$ experienced by each signal component during a time interval $T_{\mathrm{SA}}$ -on the order of a few seconds- as

$$
\tau(t) \approx \tau_{0}+\alpha t+\frac{\dot{\alpha}}{2} t^{2}+\frac{\Delta \rho(t)}{c} .
$$

In (2), $\tau_{0}$ represents the delay at the start of the observation interval; $\alpha \triangleq v_{\text {sat }-\mathbf{0}} / c$ is the Doppler coefficient, with $v_{\text {sat }-0}$ being the relative speed between the GNSS satellite and the origin of coordinates $\mathbf{0}$, e.g. the center of the trajectory described by the receiver motion, on the considered global reference frame; $c$ stands for the signal propagation speed; and $\dot{\alpha}=d \alpha / d t$ is the Doppler coefficient rate. $\Delta \rho(t)$ represents the extra propagation path travelled by the signal due to the antenna position being different from $\mathbf{0}$. This additional propagation path can be expressed as the projection of the antenna phase center position $\mathbf{p}(t)$ on the unitary vector $\mathbf{u}_{\psi}$ pointing towards the DOA of the received signal, i.e.

$$
\Delta \rho(t)=\mathbf{u}_{\psi}^{T} \mathbf{p}(t)
$$

For moving at speeds on the order of $\mathrm{m} / \mathrm{s}$, we can very well approximate expression (1), during $T_{\mathrm{SA}}$, as

$$
x(t) \approx a s\left(\left(1-\frac{f_{d}}{f_{c}}\right) t-\tau_{0}\right) \exp \left\{-j 2 \pi\left(f_{d} t+\frac{\dot{f}_{d}}{2} t^{2}+\frac{\Delta \rho(t)}{\lambda}\right)\right\}+n(t),
$$

where the effects of the extra delay due to the receiver movement with respect to $\mathbf{0}$ are only modeled as a carrier phase shift. The approximation made in Expression (4) is equivalent to taking the narrowband array assumption in a physical antenna array [2,12], and the Doppler rate effects on the code delay are neglected. In (4), we have also defined $f_{d}=\alpha f_{c}$ and $\dot{f}_{d}=\dot{\alpha} f_{c}$ as the carrier Doppler shift and carrier Doppler shift rate observed over $T_{\mathrm{SA}}$. The complex amplitude $a$-approximated as constant over $T_{\mathrm{SA}}-$ comprehends the effect of the complex antenna and the extra phase shift due to the initial constant delay $\tau_{0}$.

The signal described in (4) is amplified, filtered, down-converted and sampled by the receiver's front end. Then, the receiver cross-correlates the signal with a locally generated replica of the desired satellite signal over a time interval $T_{\text {int }}$, referred to as integration time. This approach, known as matched filtering, is optimal in the presence of additive noise and allows to estimate the delay and Doppler shift experienced by the received signal [12]. As a result, every $T_{\text {int }}$ seconds we obtain a new correlation output that, assuming a signal sampling rate sufficiently large, can be expressed as

$$
y_{k} \approx \int_{k T_{i n t}}^{(k+1) T_{i n t}} x(t) s^{*}\left(\left(1-\frac{\hat{f}_{d, k}}{f_{c}}\right) t-\hat{\tau}_{k}\right) \exp \left\{j 2 \pi \hat{f}_{d, k} t\right\} d t,
$$

where $\hat{\tau}_{k}$ and $\hat{f}_{d, k}$ are the code delay and carrier Doppler estimated by the tracking loop for the $k$ th $T_{i n t}$ interval respectively. During the signal tracking, this correlation output can be modeled as

$$
y_{k} \approx \beta_{k} \exp \left\{j \Delta \varphi_{k}\right\}+\eta_{k}
$$

where $\beta_{k}$ represents the complex amplitude of the correlator after accumulation assuming good time synchronization, i.e. $\hat{\tau}_{k} \approx \tau_{0}$, and $\hat{f}_{d, k} \approx f_{d} \cdot \eta_{k}$ is the filtered noise term. In a coherent carrier tracking scheme, e.g. a PLL, the correlation output phase $\Delta \varphi_{k}$ in (6), can be expressed as

$$
\Delta \varphi_{k}=\underbrace{\varphi_{0}+2 \pi\left(f_{d} k T_{\text {int }}+\frac{\dot{f}_{d}}{2}\left(k T_{i n t}\right)^{2}\right)}_{\begin{array}{c}
\text { Reference Doppler } \\
\text { component: } \varphi_{\text {ref }}
\end{array}}+\underbrace{\frac{2 \pi}{\lambda} \Delta \rho\left(k T_{\text {int }}\right)}_{\begin{array}{c}
\text { Relative Doppler } \\
\text { component: } \varphi_{\text {rel }}
\end{array}}-\hat{\varphi}_{k},
$$




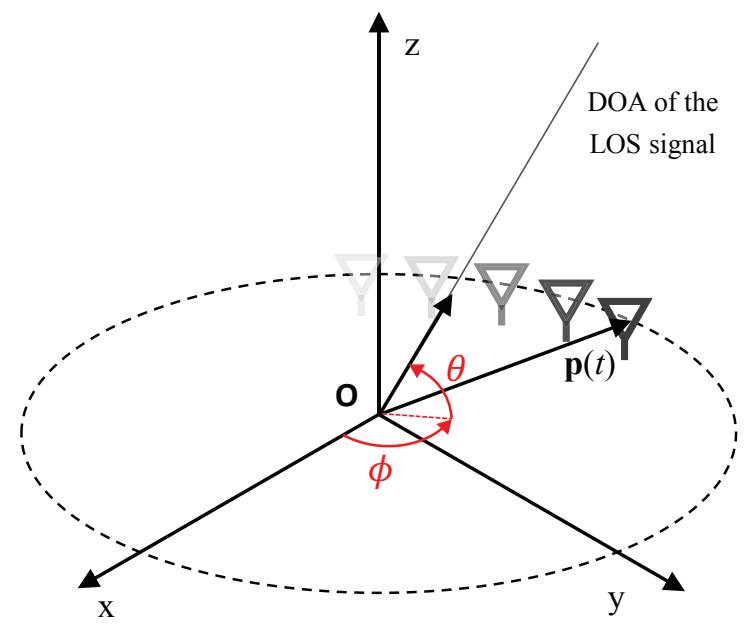

Fig. 1. Considered scenario geometry. The antenna follows a uniform circular motion along a trajectory of radius $R$. The vector $\mathbf{p}(t)$ represents the current antenna position. Lighter gray representations of the antenna are used for past positions. The angles depicted in red represent the LOS signal DOA.

where $\hat{\varphi}_{k}$ is the phase of the local replica, continuously updated by the carrier tracking loop to match the phase of the received signal. $\varphi_{0}$ is the initial phase difference with respect to the receiver's local oscillator.

In this paper we focus on the scenario where the antenna is mounted on a rotating arm as depicted in Figure 1. The antenna describes a uniform circular motion parallel to the ground plane at a rotation speed $\omega_{\text {rot }}$. Being physically attached to the mechanical arm, over a complete rotation of the arm, the antenna has also completed a $360^{\circ}$ rotation over its vertical axis, which matches the antenna boresight direction. The change in orientation of the receiving antenna with respect to the transmitting antenna, i.e. the GNSS satellite antenna, impacts the phase observed by the receiver generating the so-called phase wrap-up or phase wind-up effect $[5,9,13]$. Correcting the phase wrap-up effect is particularly important in GNSS receivers using carrier phase measurements. In the context of SA, and for satellites observed at a high elevation angles, the phase wrapup can be approximated as a constant frequency bias in the carrier measurements. In this case, the value of the bias depends only on the antenna spinning speed [13]. Nevertheless, in this paper, we consider the phase wrap-up already included within the constant frequency $f_{d}$ of the reference Doppler. For the described antenna motion, $\Delta \rho(t)$, which is directly proportional to the relative Doppler component, can be expressed as

$$
\Delta \rho(t)=R \cos (\theta) \cos \left(\omega_{\text {rot }} t+\Delta \phi\right),
$$

where $R$ is the trajectory radius; $\theta$ is the elevation angle of the received LOS signal; and $\Delta \phi=\phi_{\text {ant-0 }}-\phi$ is the azimuth difference between the LOS signal azimuth $\phi$, and the azimuth defining the antenna position at the beginning of the measurement, i.e. $\phi_{\text {ant }-0}$.

\section{REFERENCE DOPPLER ESTIMATION USING EXTENDED KALMAN FILTERING}

The use of Kalman filters (KFs) for tracking GNSS signals has received significant attention during the last years [14-17]. A KF might be thought of as a sequential minimum mean square error (MSE) estimator which exploits a statistical and dynamical model to predict and estimate the parameters of interest representing the filter states [12]. The use of KFs for carrier tracking, replacing the traditional phase-locked loops (PLLs) and frequency-locked loops, has shown several advantages under harsh tracking conditions $[14,16,17]$.

For the SA scenario under discussion, equations (7) and (8), describing the carrier phase measurements, can be linearized to develop the state model for an extended Kalman filter (EKF) [11]. The generic formulation for the EKF is sketched in Algorithm 1. We use the standard KF notation for the estimated variables -as in [17]-, i.e. ${ }_{k \mid k-1}$ for the predicted estimate at time $k$ using measurements up to time $k-1$, while ${ }_{k \mid k}$ is used for the estimated value at time $k$ using measurements up to time 


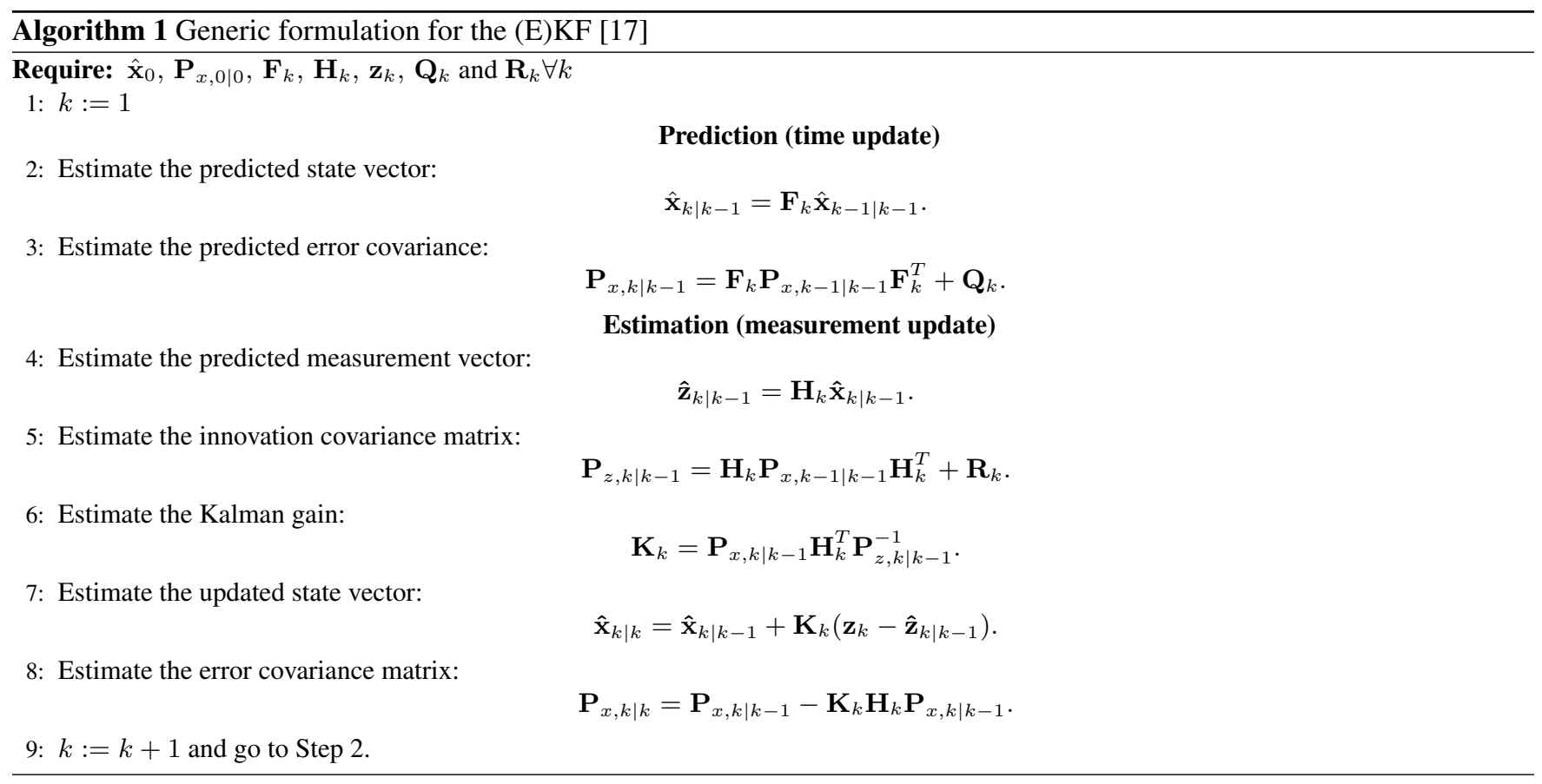

$k$. In Algorithm 1, $\hat{\mathbf{x}}$ represents the state vector estimate; $\mathbf{P}_{k}$ represents the process noise covariance matrix; $\mathbf{F}_{k}$ is the state transition matrix; $\mathbf{Q}_{k}$ is the system noise covariance matrix; the vector $\mathbf{z}_{k}$ represents the measurements with their covariance expressed by $\mathbf{R}_{k}$; and $\mathbf{H}_{k}$ stands for the observation matrix, which describes the relationship between measurements and the state vector. In general, the design of a KF requires a selection of $\mathbf{R}_{k}, \mathbf{Q}_{k}$ and $\mathbf{P}_{0 \mid 0}$. In standard KF tracking architectures, these matrices must be carefully initialized $[11,15,17]$. This selection has a major impact on the performance of the KF. While $\mathbf{R}$ can be estimated from observed measurements, the selection of $\mathbf{Q}_{k}$ is more problematic. A common approach is to use a discretization of the continuous noise model for the process noise, obtained as

$$
\mathbf{Q}_{k}=\int_{0}^{T_{i n t}} \mathbf{F}_{k} \mathbf{Q}_{c} \mathbf{F}_{k}^{T}
$$

where $\mathbf{Q}_{c}$ can be defined as a diagonal matrix with its non-zero elements being the spectral densities of each of the individual processes noises, as in [15].

We propose two new EKF-based methods to estimate carrier reference Doppler, to which we refer to as EKF1 and EKF2. The description of each method is now presented.

\section{EKF1: FLL Carrier Tracking Combined With an EKF for Reference Frequency Tracking}

In the first proposed method, the receiver uses a second-order FLL to track the received signal's carrier frequency. This FLL uses a four-quadrant arctangent discriminator, which is optimal at high and low SNRs [10]. The frequency estimated by the FLL at the time instant $t=k T_{i n t}$, can be expressed using (8) and the time derivative of (7) as

$$
\hat{f}_{k}=\underbrace{f_{d}+\dot{f}_{d} k T_{i n t}}_{f_{\text {ref }}}-\omega_{\text {rot }} \kappa \sin (\underbrace{\omega_{r o t} k T_{i n t}+\Delta \phi}_{\phi_{a}})+\eta_{f, k},
$$

with $\kappa \triangleq R \cos (\theta)$, since the trajectory's radius $R$ is fixed and known; and $\eta_{f, k}$ represents the frequency noise. $f_{\text {ref }}$ is the reference Doppler contribution -including the effect of a linear phase wrap-up-, and $\phi_{a}$ has been defined as the accumulated antenna azimuth. This frequency estimate is used as the measurement for an EKF filter, i.e. $z_{k}=\hat{f}_{k}$, defined together with the following state vector

$$
\mathbf{x} \triangleq\left[f_{\text {ref }}, \dot{f}_{d}, \kappa, \phi_{a}, \omega_{\text {rot }}\right]^{T}
$$




\begin{tabular}{cc|cc}
\hline $\mathbf{Q}_{c}$ diag. terms & $\mathbf{Q}_{c}$ values & $\mathbf{P}_{0 \mid 0}$ diag. terms & $\mathbf{P}_{0 \mid 0}$ values \\
\hline$Q_{f_{\text {ref }}}$ & $\frac{4 \pi^{3} f^{2} h_{-2}}{2 \pi^{2}} 0.1=0.0156 \mathrm{~Hz}$ & $\sigma_{f_{\text {ref }}}^{2}$ & $20^{2} \mathrm{~Hz}^{2}$ \\
$Q_{f_{d}}$ & $\frac{0.01}{2 \pi^{4}}=6.41 \cdot 10^{-6} \mathrm{~Hz}^{3}$ & $\sigma_{f_{d}}^{2}$ & $0.25 \mathrm{~Hz}^{4}$ \\
$Q_{\kappa}$ & $0.01 \mathrm{~m}^{2} / \mathrm{Hz}$ & $\sigma_{\kappa}^{2}$ & $0.25 \mathrm{~m}^{2}$ \\
$Q_{\phi_{a}}$ & $0.001 \mathrm{rad}^{2} / \mathrm{Hz}$ & $\sigma_{\phi_{a}}^{2}$ & $\pi^{2} / 3 \mathrm{rad}^{2}$ \\
$Q_{\omega_{\text {rot }}}^{2}$ & $0.001 \mathrm{rad}^{2} / \mathrm{Hz}^{3}$ & $\sigma_{\omega_{\text {rot }}}^{2}$ & $0.5^{2}(\mathrm{rad} / \mathrm{s})^{2}$ \\
\hline
\end{tabular}

Table 1. Tuning parameters selected in the system noise description for the the EKF1 case.

The process equation is formulated as

$$
\mathbf{x}_{k}=\underbrace{\left(\begin{array}{cc}
\mathbf{F}_{\text {ref }} & \mathbf{0} \\
\mathbf{0}^{T} & \mathbf{F}_{\Delta \rho}
\end{array}\right)}_{\mathbf{F}} \mathbf{x}_{k-1}+\mathbf{w}_{k},
$$

where the state transition matrix $\mathbf{F}$ has been expressed using the matrices

$$
\mathbf{F}_{\mathrm{ref}}=\left(\begin{array}{cc}
1 & T_{i n t} \\
0 & 1
\end{array}\right), \mathbf{F}_{\Delta \rho}=\left(\begin{array}{ccc}
1 & 0 & 0 \\
0 & 1 & T_{i n t} \\
0 & 0 & 1
\end{array}\right)
$$

representing the reference and relative Doppler state transition matrices, respectively. The process noise $\mathbf{w}_{k} \sim \mathcal{N}\left(\mathbf{0}, \mathbf{P}_{k}\right)$ stands for possible uncertainties or mismatches in the state model. The process noise covariance $\mathbf{P}_{k}$ is initialized to $\mathbf{P}_{0 \mid 0}$, which is defined as a diagonal matrix and updated according to Algorithm 1. The values selected for the main diagonal of $\mathbf{P}_{0 \mid 0}$ are shown in Table 1. These values were selected as the maximum expected squared error between the initial state vector, i.e. $\hat{\boldsymbol{x}}_{0}$, and true vector $\mathbf{x}$. We have defined the system noise covariance $\mathbf{Q}_{k}$ as constant using (9), with

$$
\mathbf{Q}_{c}=\operatorname{diag}\left\{\left[Q_{f_{\mathrm{ref}}}, Q_{\dot{f}_{d}}, Q_{\kappa}, Q_{\phi_{a}}, Q_{\omega_{\text {rot }}}\right]^{T}\right\}
$$

where the different $Q_{c}$ parameters, shown in Table 1, stand for the power spectral densities of the expected errors for each state in $\hat{\mathbf{x}}$. The model for $Q_{f_{\text {ref }}}$, corresponding to the reference Doppler parameter, is assuming the receiver oscillator error as the dominant error source. The parameter $h_{-2}$ depends on the type of oscillator used [15]. In our case we will set $h_{-2}=2 \cdot 10^{-20}$ $\mathrm{Hz}$, which is a reference value for a receiver's clock using an oven controlled crystal oscillator (OCXO). $Q_{\dot{f}_{d}}$, i.e. the spectral density of the reference Doppler rate, is driven by the acceleration along the line-of-sight between the origin of coordinates and the satellite. The remaining parameters in $\mathbf{Q}_{c}$ are tuned to match the expected slow variations of the state parameters. Finally, $\omega_{\text {rot }}$ will not remain exactly constant in a real measurement scenario. Therefore $Q_{\omega_{\text {rot }}}>0 . Q_{\kappa}$ and $Q_{\phi_{a}}$ account for the small evolution of the observed signal's DOA.

In the EKF1 approach there is only one measurement available: the frequency estimated by the FLL, i.e. $z_{k}=\hat{f}_{k}$. The measurement model is described as

$$
z_{k}=\mathbf{H}_{k} \mathbf{x}_{k}+v_{k}
$$

where $v_{k} \sim \mathcal{N}\left(0, \sigma_{\hat{f}_{k}}^{2}\right)$ is the measurement's noise. The linearized observation matrix in equation (15), i.e. $\mathbf{H}_{k} \in \mathbb{R}^{1 \times 5}$, is defined as

$$
\mathbf{H}_{k}=\left[1,0,-\omega_{r o t, k \mid k-1} \sin \left(\phi_{a, k \mid k-1}\right),-\omega_{r o t, k \mid k-1} \kappa_{k \mid k-1} \cos \left(\phi_{a, k \mid k-1}\right),-\kappa_{k \mid k-1} \sin \left(\phi_{a, k \mid k-1}\right)\right] .
$$

We assume that the noise variance of the FLL output is mainly caused by thermal noise. This assumption is reasonable for the motions considered in this work, and allows us to neglect the dynamic stress error. We have selected the measurement $z_{k}$ noise variance to match the thermal noise variance [10], i.e. 


$$
R_{k}=\sigma_{\hat{f}_{k}}^{2} \approx \frac{1}{2 C / N_{0} T_{i n t}}\left(1+\frac{1}{2 C / N_{0} T_{i n t}}\right),
$$

where $C / N_{0}$ is the carrier-to-noise density of the received signal at the instant $k$. In practice, the true $C / N_{0}$ is not known and shall be estimated by the receiver. In this paper, we have assumed that the true $C / N_{0}$ remains approximately constant during the entire signal observation time.

\section{EKF2: Carrier Phase Tracking using an EKF for Ultra-tight Integration with the Antenna Motion Model}

In the second proposed method, carrier phase tracking is performed using an EKF that integrates, in an ultra-tightly scheme [11], the information provided by the antenna motion model, with its effects on the observed phase described by equation (8). The state vector for this EKF is defined as

$$
\mathbf{x} \triangleq\left[\varphi_{\mathrm{ref}}, f_{\mathrm{ref}}, \dot{f}_{d}, \kappa, \phi_{a}, \omega_{\text {rot }}, \beta\right]^{T},
$$

where $\varphi_{\text {ref }}$ represents the accumulated phase due to the reference Doppler, and $\beta$ is the amplitude of the prompt correlator output [10], which was introduced in equation (6). The process equation is formulated as shown in equation (12), with the state transition matrix $\mathbf{F}$ expressed now using the following matrices

$$
\mathbf{F}_{\text {ref }}=\left(\begin{array}{ccc}
1 & 2 \pi T_{\text {int }} \pi T_{\text {int }}^{2} \\
0 & 1 & T_{\text {int }} \\
0 & 0 & 1
\end{array}\right), \mathbf{F}_{\Delta \rho}=\left(\begin{array}{cccc}
1 & 0 & 0 & 0 \\
0 & 1 & T_{\text {int }} & 0 \\
0 & 0 & 1 & 0 \\
0 & 0 & 0 & 1
\end{array}\right) .
$$

The values selected to initialize $\mathbf{P}_{k}$, i.e. $\mathbf{P}_{0 \mid 0}$, are shown in Table 2 . We have defined the system's noise covariance $\mathbf{Q}_{k}$ as constant using expression (9), with

$$
\mathbf{Q}_{c}=\operatorname{diag}\left\{\left[Q_{\varphi_{\mathrm{ref}}}, Q_{f_{\mathrm{ref}}}, Q_{\dot{f}_{d}}, Q_{\kappa}, Q_{\phi_{a}}, Q_{\omega_{\text {rot }}}, Q_{\beta}\right]^{T}\right\} .
$$

Most of the parameters in $\mathbf{Q}_{c}$ are defined in the same way as in expression (14) for EKF1. $Q_{\beta}$ accounts for any slow drift on the observed signal amplitude, which the process model assumes constant. $Q_{\varphi_{\text {ref }}}$ stands for the power spectral density of the expected error on the reference Doppler phase, which we assume that is mostly caused by the receiver clock error $[15,17]$. The parameters selected for $\mathbf{Q}_{c}$ are shown in Table 2. The model for $Q_{\varphi_{\text {ref }}}$ is defined as a function of the parameter $h_{0}=8 \cdot 10^{-20}$ $\mathrm{s}$, for a receiver driven by an OCXO [15].

\begin{tabular}{cc|cc}
\hline $\mathbf{Q}_{c}$ diag. terms & $\mathbf{Q}_{c}$ values & $\mathbf{P}_{0 \mid 0}$ diag. terms & $\mathbf{P}_{0 \mid 0}$ values \\
\hline$Q_{\varphi_{\text {ref }}}$ & $\pi f^{2} h_{0}=3.898 \cdot 10^{-3} \mathrm{rad}^{2} / \mathrm{Hz}$ & $\sigma_{\varphi_{\text {ref }}}^{2}$ & $\pi^{2} / 3 \mathrm{rad}^{2}$ \\
$Q_{f_{\text {ref }}}$ & $\frac{4 \pi^{3} f^{2} h_{-2}}{2 \pi^{2}}=0.156 \mathrm{~Hz}$ & $\sigma_{f_{\text {ref }}}^{2}$ & $10^{2} \mathrm{~Hz}^{2}$ \\
$Q_{\dot{f}_{d}}$ & $\frac{5}{2 \pi^{4}} \mathrm{~Hz}^{3}$ & $\sigma_{\dot{f}_{d}}^{2}$ & $4 \mathrm{~Hz}^{4}$ \\
$Q_{\kappa}$ & $0.01 \mathrm{~m}^{2} / \mathrm{Hz}$ & $\sigma_{\kappa}^{2}$ & $0.25 \mathrm{~m}^{2}$ \\
$Q_{\phi_{a}}$ & $0.001 \mathrm{rad}^{2} / \mathrm{Hz}$ & $\sigma_{\phi_{a}}^{2}$ & $\pi^{2} / 3 \mathrm{rad}^{2}$ \\
$Q_{\omega_{\text {rot }}}^{2}$ & $0.001 \mathrm{rad}^{2} / \mathrm{Hz}^{3}$ & $\sigma_{\omega_{\text {rot }}}^{2}$ & $0.05^{2}(\mathrm{rad} / \mathrm{s})^{2}$ \\
$Q_{\beta}$ & $\left(200 T_{\text {int }}\right)^{2}$ & $\sigma_{\beta}^{2}$ & $10^{2}$ \\
\hline
\end{tabular}

Table 2. Tuning parameters selected in the system noise description for the the EKF2 case.

The EKF2 measurement vector has been defined as $\mathbf{z}_{k}=\left[\Re\left\{y_{k}\right\}, \Im\left\{y_{k}\right\}\right]^{T}$, i.e. the in-phase and quadrature components of the receiver's prompt correlator output. The linearized observation matrix describing the measurement model has been computed as ${ }^{1}$

\footnotetext{
${ }^{1}$ The vector differential operator is defined as $\nabla=\left[\partial / \partial x_{1}, \ldots, \partial / \partial x_{n}\right]$
} 


$$
\mathbf{H}_{k}=\left.\nabla \mathbf{h}_{k}\left(\mathbf{x}_{k}\right)\right|_{\mathbf{x}_{k}=\hat{\mathbf{x}}_{k \mid k-1}},
$$

where we have defined the measurement transition function $\mathbf{h}_{k}\left(\mathbf{x}_{k}\right)$ as

$$
\mathbf{h}_{k}\left(\mathbf{x}_{k}\right)=\left[\begin{array}{l}
\beta_{k} \cos \left(2 \pi\left(\varphi_{\mathrm{ref}, k}-\hat{\varphi}_{\mathrm{ref}, k-1 \mid k-1}+\kappa_{k} \cos \left(\phi_{a, k}\right)-\hat{\kappa}_{k-1 \mid k-1} \cos \left(\hat{\phi}_{a, k-1 \mid k-1}\right)\right)\right) \\
\beta_{k} \sin \left(2 \pi\left(\varphi_{\mathrm{ref}, k}-\hat{\varphi}_{\mathrm{ref}, k-1 \mid k-1}+\kappa_{k} \cos \left(\phi_{a, k}\right)-\hat{\kappa}_{k-1 \mid k-1} \cos \left(\hat{\phi}_{a, k-1 \mid k-1}\right)\right)\right)
\end{array}\right] .
$$

The covariance matrix of the measurements $\mathbf{z}_{k}$ is therefore characterized as

$$
\mathbf{R}_{k}=\frac{\sigma_{\eta, k}^{2}}{2} \mathbf{I}
$$

where $\sigma_{\eta, k}^{2}$ is the variance of the noise at the correlator output samples, which is a function of the observed $C / N_{0}$ and $T_{\text {int }}$. In practice, the receiver must estimate the value for $\sigma_{\eta, k}^{2}$. In this paper we have approximated it as constant, given an initial guess for the $C / N_{0}$.

\section{REFERENCE DOPPLER ESTIMATION METHODS COMPARISON}

In this section, we compare the results of four different methods to estimate the reference Doppler: the linear interpolation method using LSE; the reference static antenna method; and the two EKF-based methods that we propose, i.e. EKF1 and EKF2. The first two methods have been briefly described in the Introduction section, and more details can be found in $[5,7,18,19]$. The EKF1 and EKF2 methods have been thoroughly described in the previous section.

In this paper, we consider only the Global Positioning System (GPS) L1 Coarse Acquisition (C/A) signal [10], but we shall emphasize that methods discussed can be used for any GNSS signal. The received signals were processed using an NSL RF stereo front end [20] with an output sampling frequency of 6.5 MHz. The front end's raw output was recorded for processing offline. The four estimation methods have been implemented by modifying the MATLAB's Kai-Borre software defined GPS receiver [21].

Our comparison is divided into two parts. In the first part, we used synthetic GPS signal generated using a Spirent GSS8000 simulator [22]. The use of Spirent simulator provides precise control over the reception scenario, and therefore, it is particularly useful to validate the proposed EKF methods, and to verify their implementation. The simulator allows us to accurately define the desired receiver motion. We used this feature to simulate an ideal uniform circular trajectory of radius $R=0.9 \mathrm{~m}$ and an angular speed of $\omega_{\text {rot }}=0.5 \mathrm{rev} / \mathrm{s}$ or $30 \mathrm{rpm}$. Simultaneously, the antenna was simulated to spin around the vertical axis at the same angular speed. In the second part of our comparison, we used real GPS signal. To collect this signal, we used a GPS antenna mounted on a mechanical rotating arm to approximately replicate the simulated antenna motion. As described in $[6,18]$, SA techniques imply very long coherent integration times, requiring a highly stable clock. Therefore, for our tests we used an external OCXO to drive the front end.

In each part we will study:

1. How accurately the different methods can estimate the reference Doppler.

2. The impact of the reference Doppler estimate on the SA beamforming and DOA estimation techniques.

To do the latter, we have implemented the beamscan DOA estimation algorithm [7,12]. The beamscan algorithm is the counterpart of the delay-and-sum beamforming algorithm. In single-source scenarios, the DOA map computed using beamscan, i.e. an estimate of the power received as a function of the DOA angle, is equivalent to the gain pattern synthesized by the delayand-sum algorithm. Furthermore, beamscan is the simplest DOA algorithm and it is independent of the channel statistics, which makes it particularly robust against severe multipath [12]. Below, we summarize the main results obtained in each part. We also provide more details on the setup used in each specific case.

The observed azimuth angle $\phi$ is measured with respect to the rotating arm's position when the first measurement -used in the DOA estimation- was taken. This is considered the $\phi=0^{\circ}$ azimuth angle. Matching $\phi=0^{\circ}$ to the true north direction 


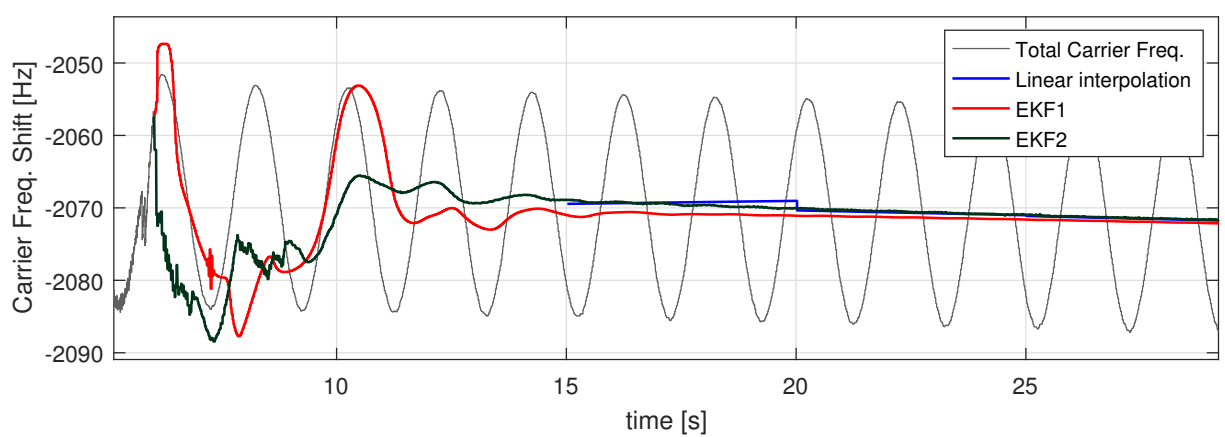

(a)

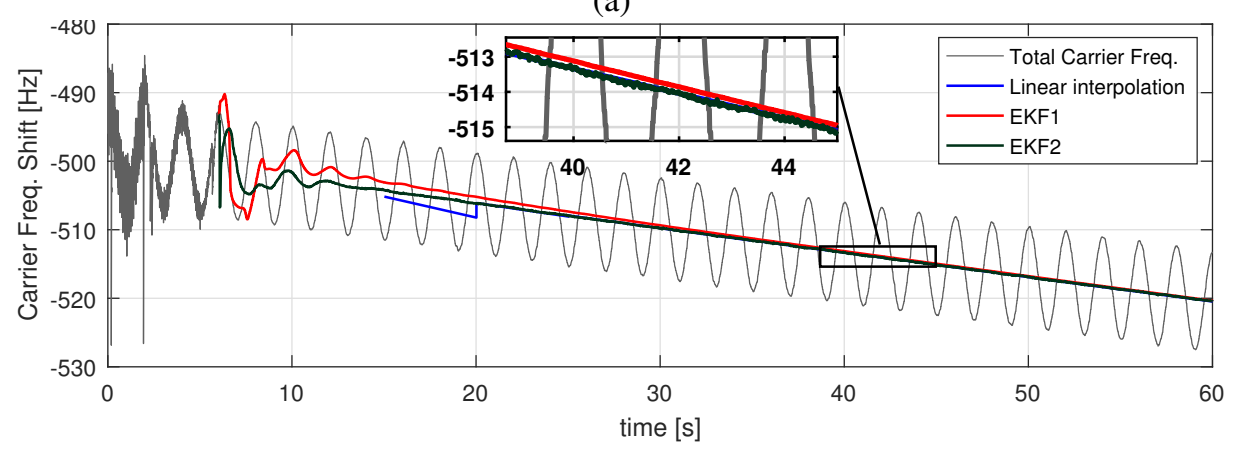

(b)

Fig. 2. Estimated reference Doppler for (a) PRN 22, and (b) PRN 23 in the Spirent simulated scenario. We depicted the total carrier frequency, tracked by an FLL, in gray. The reference Doppler estimates are depicted with colored lines. For each method, the beginning of the line marks the first estimation.

when recording real GPS signal using the rotating arm can be challenging, if no additional sensor to track the arm's position is used. Unfortunately, when the data used in this paper were recorded, our arm did not have any sensor implemented capable of such thing. Not having $\phi=0^{\circ}$ corresponding to the true north direction will add a constant bias to the estimated azimuth. This bias is just the difference between the north direction and our arbitrarily selected $\phi=0^{\circ}$. This difference only implies a circular shift on the computed DOA map in the azimuth direction, without introducing any distortion. For consistency we followed the arbitrary $\phi=0^{\circ}$ definition approach also for the simulated signal. We shall highlight this as the reason why DOA maps for the same satellite, but corresponding to different times will show different $\phi$ values.

\section{Simulations using Synthetic Signal}

In this section we describe the results obtained using the synthetic GPS signal generated by our GSS8000 Spirent simulator. Neither antenna gain, nor polarization mismatch effect [9] were simulated, and therefore, no phase wrap-up effect should be observed. The simulator's RF output was amplified using a LNA before feeding the NSL RF front end to record $60 \mathrm{~s}$ of signal. The simulation scenario was purposely selected to match the time and location of the real measurement campaign, which will be discussed in the following section. Among the visible satellites, we selected PRN 23, observed with an elevation $\theta \approx 63^{\circ}$, and PRN 22, observed with an elevation $\theta \approx 23^{\circ}$. The reason to describe the elevation as approximate is because it slightly changes during the considered observation time.

For the linear interpolation method, $5 \mathrm{~s}$ of carrier frequency estimates were used as input for the LSE. The LSE output was used to interpolate the reference Doppler every $5 \mathrm{~s}$. The reference static antenna method has not been considered for this simulation scenario. Figure 2 shows the reference Doppler estimation results obtained with the remaining three methods, for two different satellites. Figure 2.(a) shows only the first $30 \mathrm{~s}$ of carrier tracking. The total carrier frequency is noisier at the start of the recording because the receiver reduced the carrier tracking bandwidth -in 2 steps- until it started the reference Doppler estimation. The observed oscillations were caused by the relative Doppler component, with their period matching the antenna rotation period. Abrupt changes, in the shape of small steps, are observed when using the linear interpolation method. These steps appear every time the LSE output is updated. EKF1 and EKF2 methods take approximately $10 \mathrm{~s}$ to converge. However, 


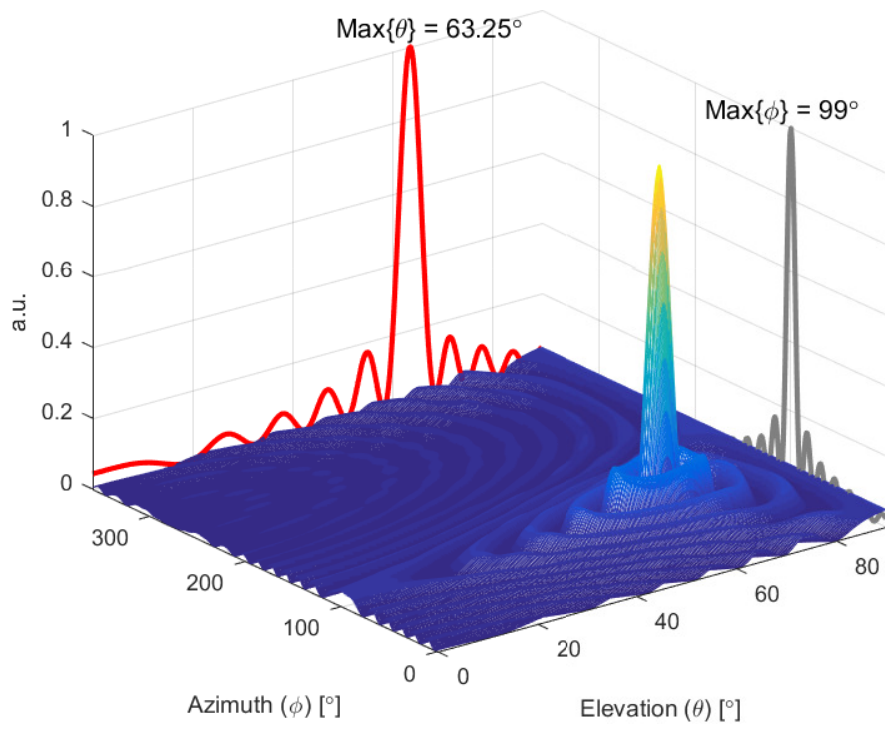

Fig. 3. DOA map for PRN 23 in the Spirent simulated scenario, with reference Doppler compensated using EKF2 method. The first input sample for the beamscan algorithm corresponds to $t=40 \mathrm{~s}$.

the zoomed view in Figure 2.(b) shows a small offset between the EKF1 and the two other methods. The offset can be even observed once the EKF1 has been operating for several seconds. It is not clear what is causing this offset.

In Figure 3, we show the DOA map computed using beamscan for PRN 23. The beamscan input are prompt correlator output samples after their compensation for the reference Doppler, using EKF2 method, over one complete circular trajectory, i.e. during a rotation period $T_{\mathrm{SA}}$. The projections of the DOA map maximum into the $\mathrm{XY}$ and $\mathrm{YZ}$ planes have been depicted in red and gray in Figure 3. These projections or "slices" are useful to evaluate the distortion on the DOA map computed versus the ideal case. We define this ideal case as the DOA map computed for an ideal physical uniform circular array (UCA) with $N=T_{\mathrm{SA}} / T_{\text {int }}$ elements, and known the DOA of the signal. In Figure 4 we show these elevation and azimuth "slices" for the PRN 23's DOA map, in the same case, after using different methods to compensate the reference Doppler. The ideal UCA case -labelled "Theoretical"- is shown as benchmark to measure distortions and offsets on the obtained DOA map. Interestingly, a significant error is observed in the estimated elevation $\hat{\theta}$ for the EKF1 method, with a larger secondary peak at $\theta \approx 70^{\circ}$. EKF2 shows a smaller error in the estimated elevation, while the linear interpolation method closely matches the ideal case. However, the linear interpolation method shows the largest error in its azimuth estimate.

\section{Real Measurements using ESPLAB's Rotating Arm}

Real GPS L1 signal measurements were recorded on Nov 28th, 2016, starting at 14h49 GMT+1 from the rooftop of the Microcity building in Neuchâtel (Location: 46 59'51.3276” N, 656'44.916” E). An Antcom 42GNSSA-RL-XT-1 antenna [23] was mounted on a rotating arm built at the ESPLAB in collaboration with the University of Balearic Islands. The rotating arm was supported by a fully-adjustable tripod as shown in Figure 5. The location was chosen as an open-sky scenario with little multipath presence expected. The antenna position was adjusted for a radius of $0.9 \mathrm{~m}$, measured from the arm rotation axis to the antenna phase center. Vertical oscillations of the antenna during the arm rotation were minimized by carefully counterbalancing the loaded arm. The nominal antenna rotation speed $\omega_{\text {rot }}$ was adjusted to $0.5 \mathrm{rev} / \mathrm{s}$. However, the arm's maximum $\omega_{\text {rot }}$ is 1.66 rev/s, i.e. $100 \mathrm{rpm}$, which is caused by the RF rotary joint mechanical limitations. The RF signal from the antenna is passed through a RF rotary joint to reach the NSL RF front end. We recorded $90 \mathrm{~s}$ of raw front end output samples. An additional Antcom antenna was connected to the NSL front end secondary channel. This antenna remained static at $2.5 \mathrm{~m}$ distance from the rotating arm rotation's axis. The carrier Doppler observed by this antenna has been used by the reference static antenna method. The antenna was placed at sufficient distance to prevent any blockage or interference from the rotating arm.

In Figure 6 we show the reference Doppler estimated by the different methods for PRNs 7 and 23. The linear interpolation method seems to perform poorly, and in some time intervals the difference with respect to the actual reference Doppler can be 

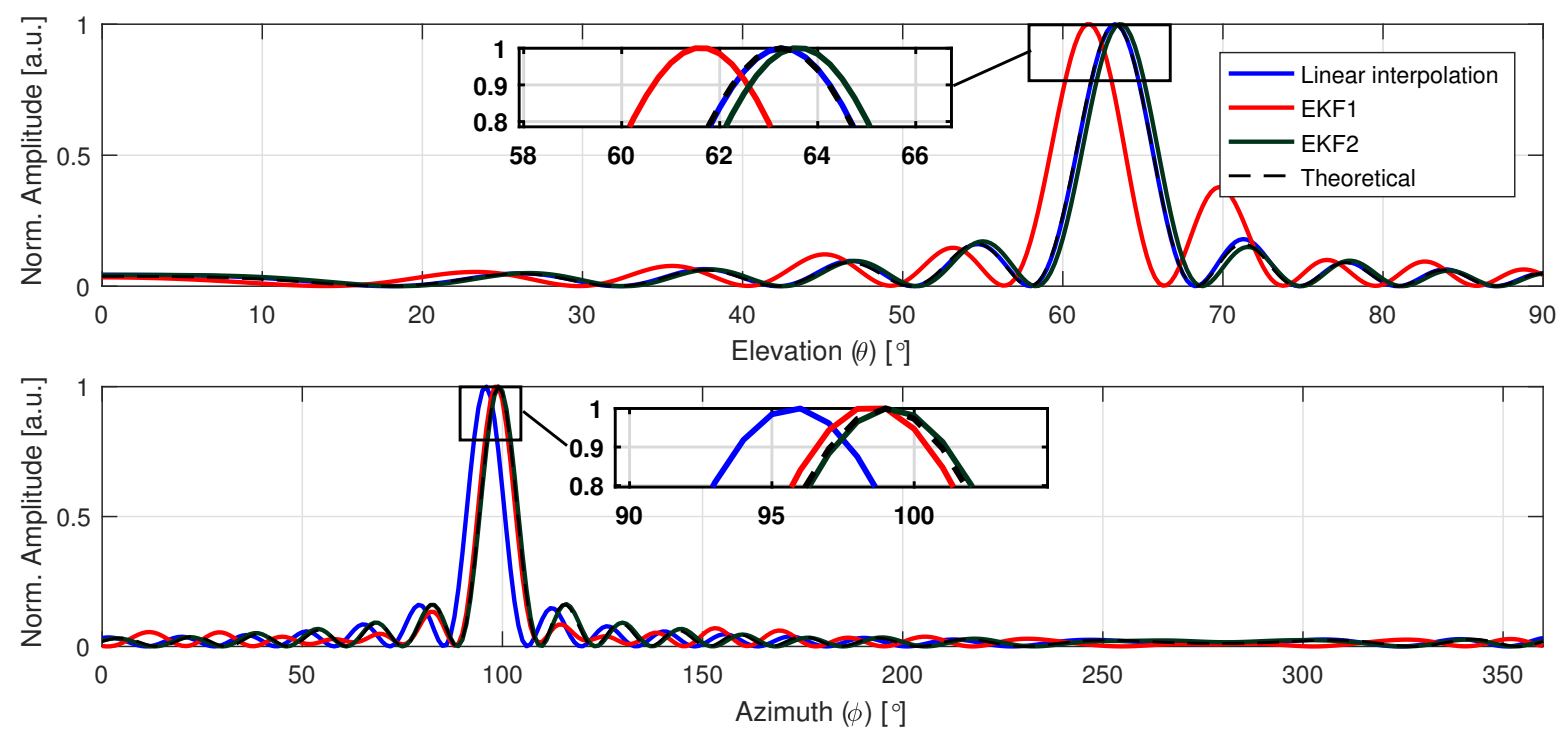

Fig. 4. DOA map peak's projections in elevation (top) and azimuth (bottom) for PRN 23 in the Spirent simulated scenario, using different reference Doppler compensation methods.

larger than $1 \mathrm{~Hz}$. EKF2 estimates show some residual periodic oscillation, matching the antenna rotation period. EKF1 appears to provide the smoothest estimate. The variance of the reference Doppler estimates provided by the static antenna method depends on the noise bandwidth of the carrier tracking loop used to track the signal received by the static antenna. In this case, we used a second-order FLL with $1 \mathrm{~Hz}$ bandwidth. The phase-wrap up effect cannot be compensated with the static antenna method. Thus, we have manually added a frequency offset, different for each satellite, to their reference Doppler estimates to match the average trend observed by the other methods.

In Figure 7 we show the DOA maps computed using the beamscan algorithm for PRN 23 (top row) and PRN 7 (bottom row) for different reference Doppler estimation methods, starting at time $t=52 \mathrm{~s}$ in the recorded measurements. The reference Doppler estimation around $t=52 \mathrm{~s}$ has been magnified in Figure 6. The linear interpolation and the EKF1 methods were used to obtain the results shown, respectively, in Figure 7.(a-b). Similar results for PRN 7, but this time with the EKF2 method instead of EKF1, are presented in Figure 7.(c-d). The instant $t=52 \mathrm{~s}$ was deliberately chosen because of the observed deviation shown by the linear interpolation method estimates from what appears to be the actual reference Doppler, for both PRNs under consideration.

As in the simulation scenario case previously discussed, we present the DOA map "slices" for PRN 23 at two different time instants, namely $t_{1}=62 \mathrm{~s}$ in Figure 8 and $t_{2}=52 \mathrm{~s}$ in Figure 9. We recall these "slices" corresponding to the projections in elevation and azimuth of the DOA map's peak. Figure 6.(b) shows that in $t_{1}=62 \mathrm{~s}$, the reference Doppler estimates from the different methods closely match, including the linear interpolation method. Hence, in Figure 8, all the methods display a very similar behavior. Nonetheless, the EKF1 and the static antenna methods show a small bias in the elevation estimate when compared to the ideal DOA shape. Figure 9, on the other hand, exemplifies the case where the linear interpolation method is significantly off from the actual reference Doppler, and DOA map is considerably distorted.

In Figure 10 and Figure 11 we summarize the received signal elevation error over time for PRN 23 and PRN 7, respectively, when using the different studied methods. The elevation error is shown for an interval of $30 \mathrm{~s}$, starting from $t=40 \mathrm{~s}$, in order to ensure that EKF1 and EKF2 had time to previously converge. We considered the satellite elevation obtained from the 


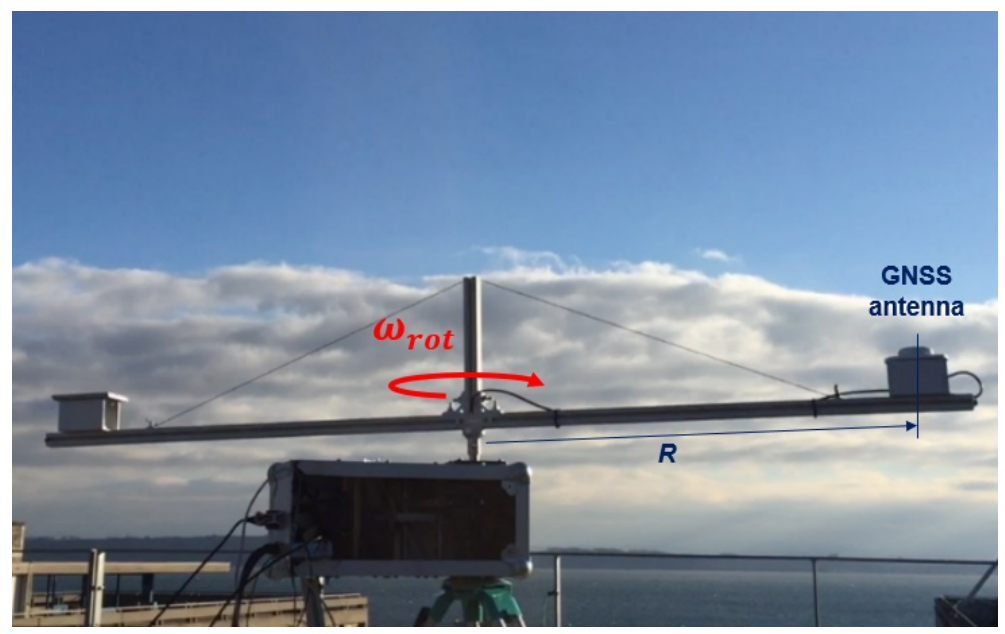

Fig. 5. Picture of the ESPLAB's mechanical rotating arm setup when the real signal measurements were taken.

\begin{tabular}{ccc}
\hline & PRN 23 & PRN 7 \\
\hline Linear interpolation & $4.704^{\circ}$ & $37.368^{\circ}$ \\
Ref. static antenna & $0.286^{\circ}$ & $2.808^{\circ}$ \\
EKF1 & $0.323^{\circ}$ & $2.808^{\circ}$ \\
EKF2 & $1.075^{\circ}$ & $3.274^{\circ}$ \\
\hline
\end{tabular}

Table 3. MSE of the LOS signal elevation estimate $(\hat{\theta})$ for PRNs 23 and 7, obtained using the beamscan DOA estimaton algorithm after removing the reference Doppler with the different methods.

navigation solution computed by the receiver as the true satellite elevation. Table 3 summarizes the mean square error (MSE) in the estimated elevation for the methods considered in this paper.

Finally, in Figure 12 we show the arm rotation speed estimated by the EKF1 and EKF2 methods using PRN 23 and PRN 7 independently. The rotation speed estimate obtained as a side product of the LSE when using the linear interpolation method is also shown. After converging, the results in Figure 12 show how the actual rotation speed oscillates around the nominal $0.5 \mathrm{rev} / \mathrm{s}$ value. This can be explained by the mild wind conditions during the measurement recording, that might well impact arm's instantaneous rotation speed. An error in the assumed rotation speed implies an error on the assumed trajectory of the antenna, required by the DOA estimation algorithm. This kind of error has shown to severely impact the spatial filtering when using GNSS SA techniques, as described in [8]. This effect might be additionally contributing to the poor results for the linear interpolation method at $t=52 \mathrm{~s}$. Indeed, Figure 12 shows that at that time instant, the rotation speed diverges from $0.5 \mathrm{rev} / \mathrm{s}$ ( $\pi$ $\mathrm{rad} / \mathrm{s}$ in the plot), while for $t=62 \mathrm{~s}$ the estimated rotation speed is much closer to that value.

\section{CONCLUSION}

SA techniques have the potential to improve the performance of GNSS receivers in the presence of interfering signals. This paper has proposed two new methods, based on the similar principle, that can enable the use of SA techniques in a wider range of receivers.

The comparison of the four considered methods has stressed how critical it is to accurately compensate the reference Doppler before attempting any spatial filtering in the context of SA, in this case DOA estimation. Our results confirm that small estimation errors, i.e. on the order of $1 \mathrm{~Hz}$, can severely bias the DOA estimation.

The two methods proposed in this paper, i.e. EKF1 and EKF2, have proven capable of using the antenna motion model to successfully track the reference Doppler. They solve the main shortcoming of the linear interpolation method, i.e. selecting 


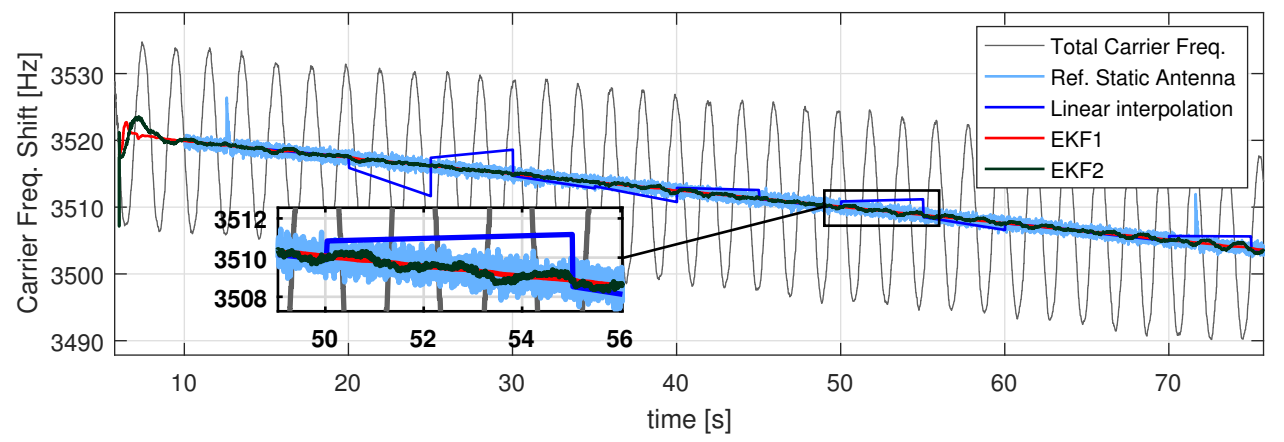

(a)

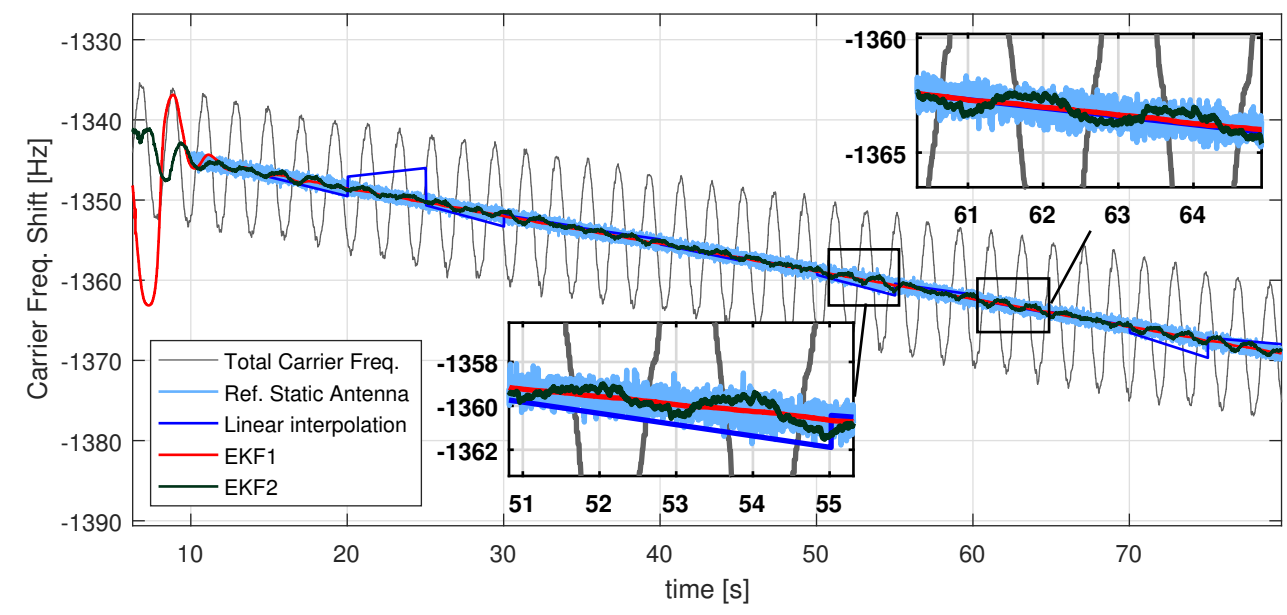

(b)

Fig. 6. Estimated reference Doppler for (a) PRN 7, and (b) PRN 23 using real measurements. Again, the total carrier frequency tracked is depicted the in gray. The reference Doppler estimates are depicted with colored lines. For each method, the beginning of the line marks the first estimation. 


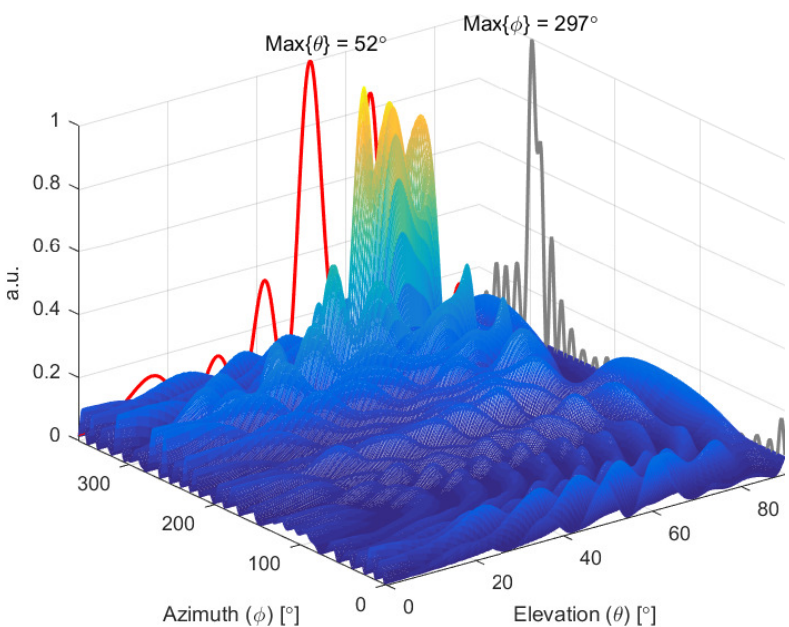

(a)

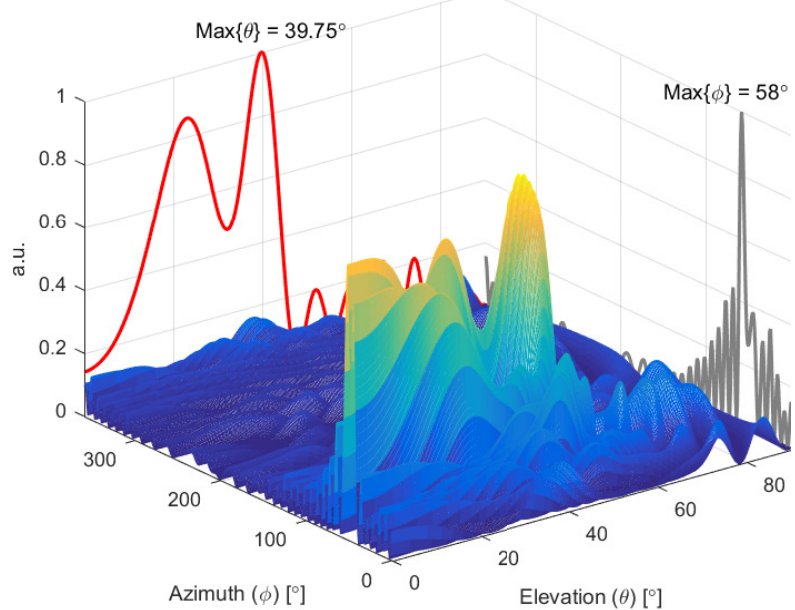

(c)

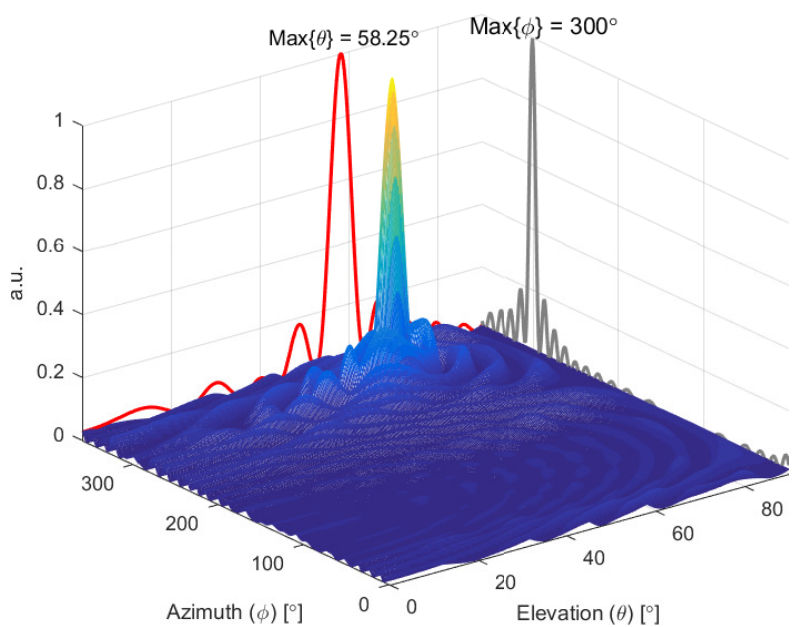

(b)

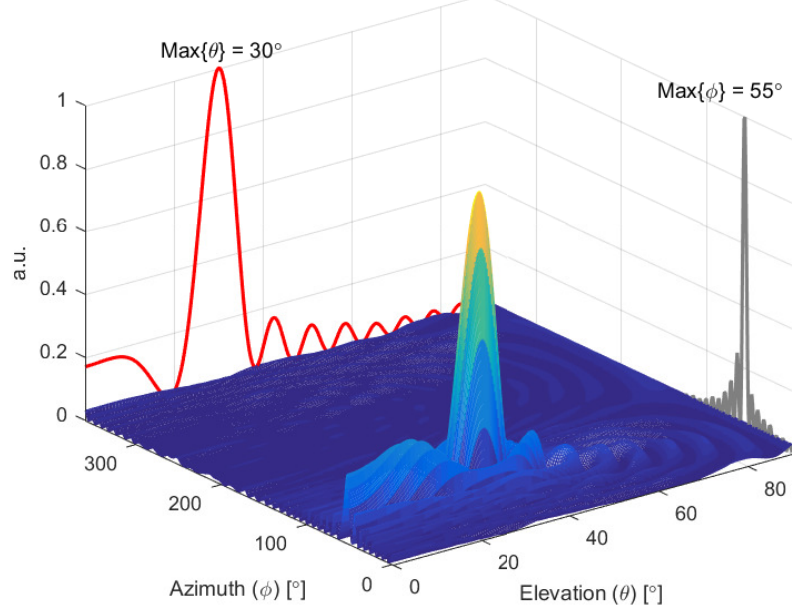

(d)

Fig. 7. DOA maps computed using the recorded real signal, starting at the instant $t=52 \mathrm{~s}$. (a) PRN 23 and linear interpolation; (b) PRN 23 and EKF1; (c) PRN 7 and linear interpolation; and (d) PRN 7 with EKF2. 

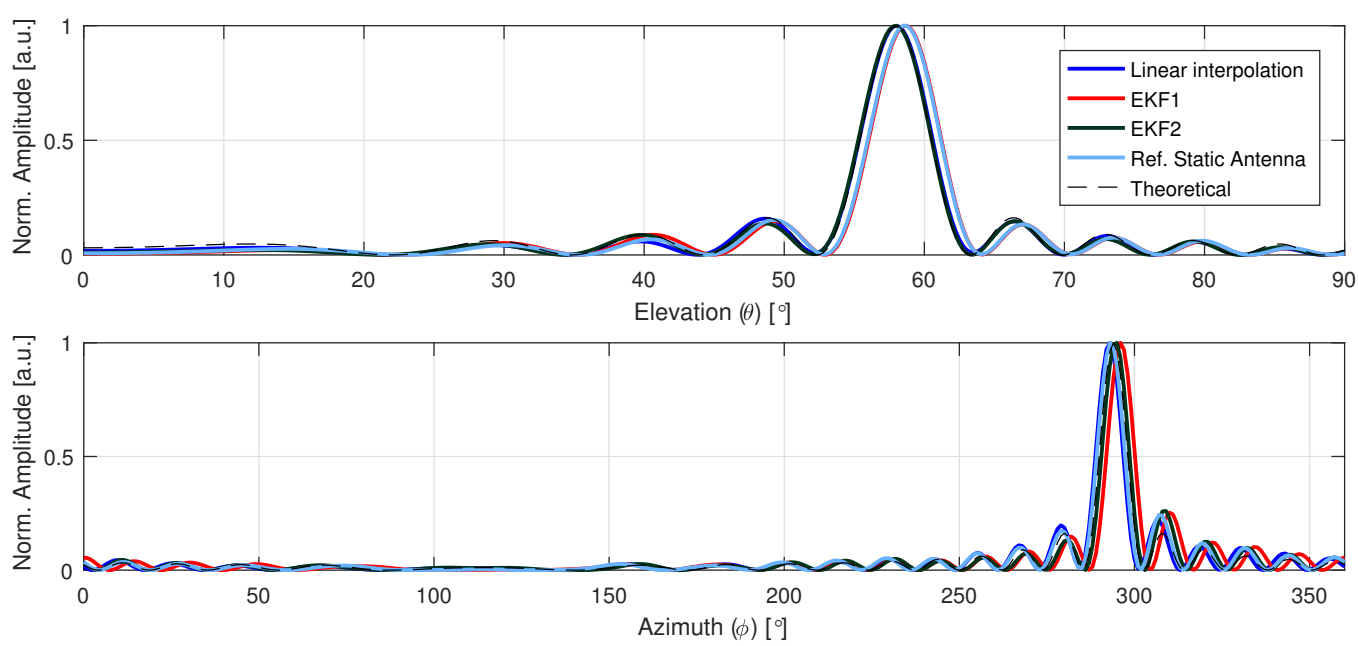

Fig. 8. DOA map peak's projections in azimuth (top) and elevation (bottom) for PRN 23 for the recorded real signal, starting at $t_{1}=62 \mathrm{~s}$, using different reference Doppler compensation methods.
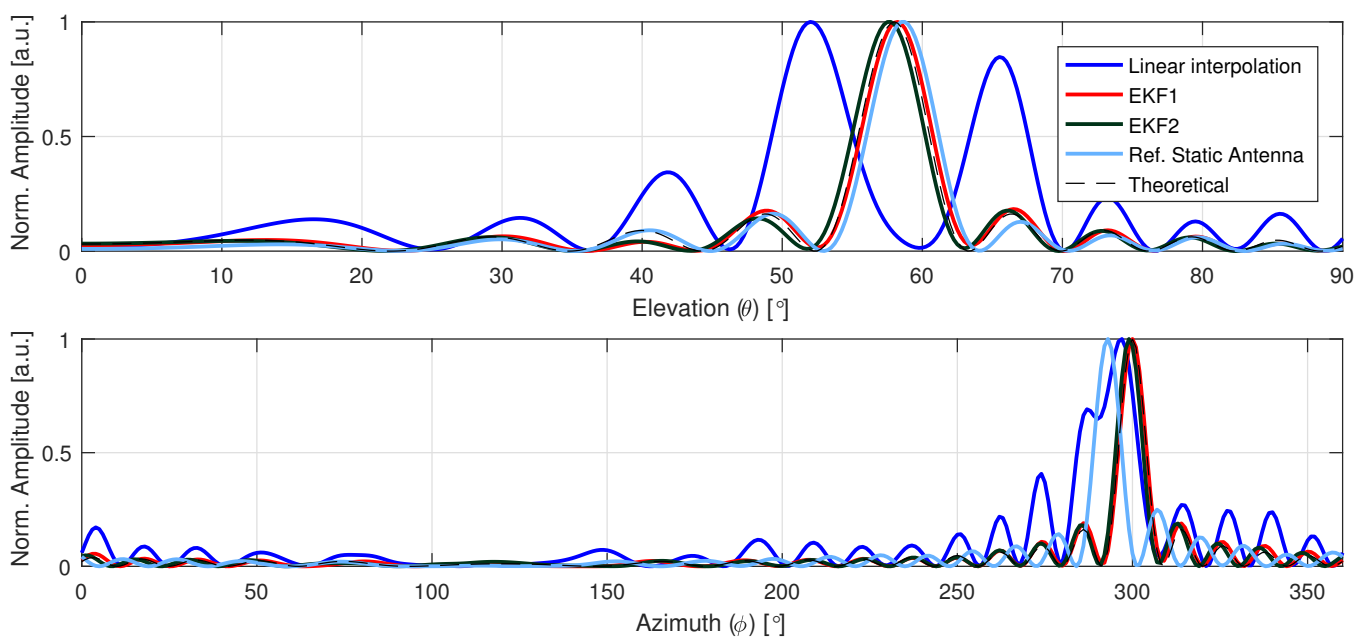

Fig. 9. DOA map peak's projections in azimuth (top) and elevation (bottom) for PRN 23 for the recorded real signal, starting at $t_{2}=52 \mathrm{~s}$, using different reference Doppler compensation methods.

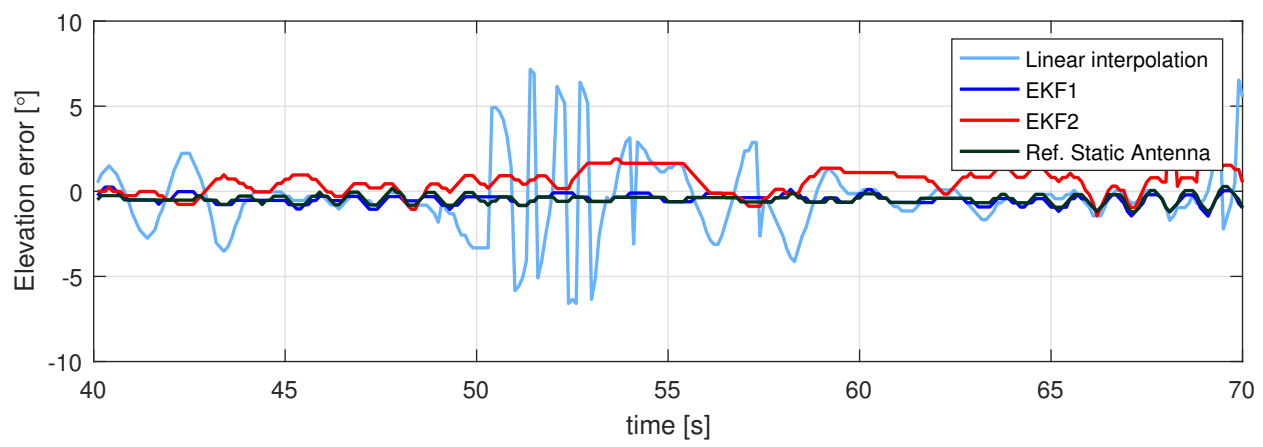

Fig. 10. Estimated elevation error for PRN 23, using the beamscan algorithm, for the different reference Doppler estimation methods. 


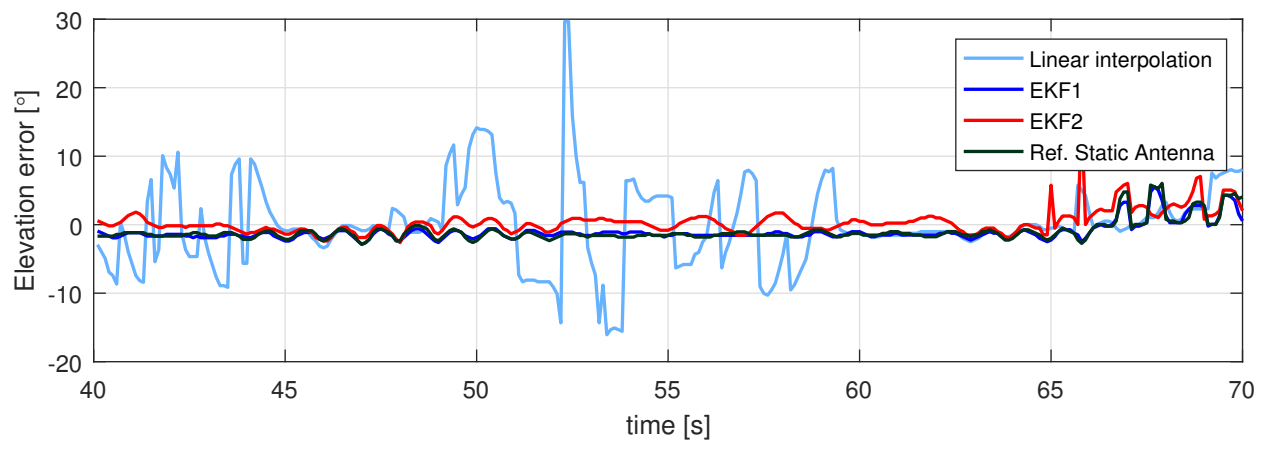

Fig. 11. Estimated elevation error for PRN 7, using the beamscan algorithm, for the different reference Doppler estimation methods.

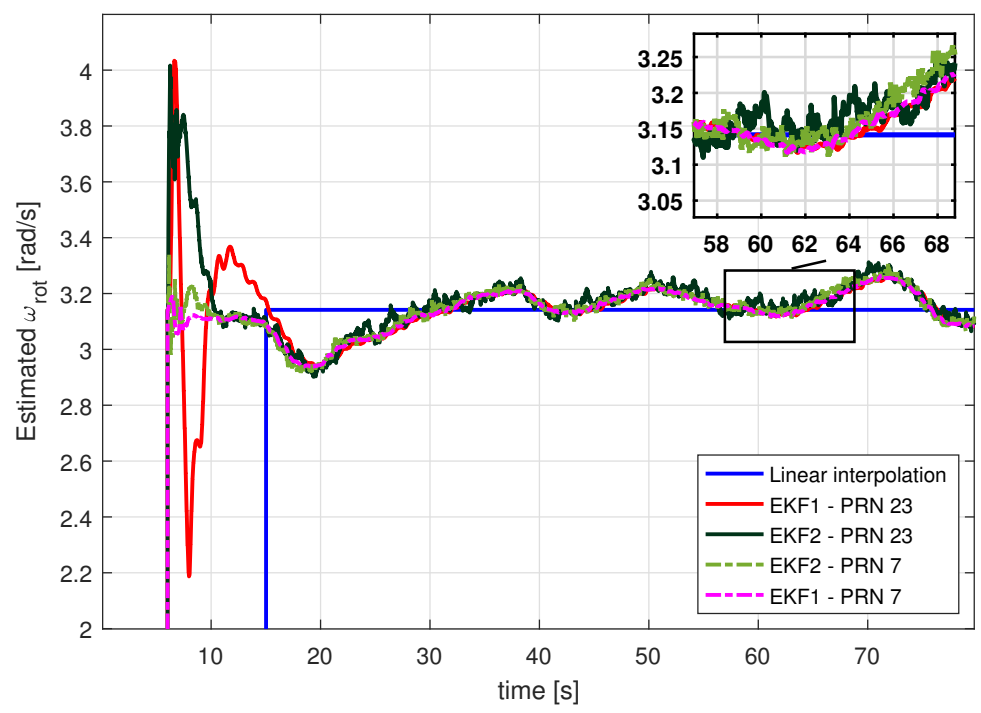

Fig. 12. Estimated antenna rotation speed $\left(\omega_{\text {rot }}\right)$ for PRN 23 and PRN 7 
the right input data size for the LSE; and there is no need for an additional hardware and signal processing, like in the case of using the reference static antenna method. In many GNSS platforms, such as in drones or hand-held devices, the limitation of requiring an static antenna cannot be easily overcome. Slow carrier frequency deviations, e.g. caused by receiver's clock error, can also be tracked as they are included within the reference Doppler, and therefore potentially corrected. In addition, the phase wrap-up can also be compensated by the EKF1 and EKF2 methods, while it is simply not possible to do so with the reference static antenna method. According to the results, EKF1 has been found to be more significantly robuster than EKF2. This can be particularly important in scenarios with severe -non zero-mean- multipath, where the carrier phase or frequency measured model corresponds to the sum of the different multipath components. The less the received signal matches the model assumed, the worst the performance of the EKF, which might even diverge. In the case of the EKF2 this leads to loss of lock situations.

Finally, we want to emphasize the importance of the EKF tuning on the performance of the proposed methods. In this paper, we have used an heuristic approach for selecting the tuning parameters, i.e. the $\mathbf{Q}_{k}, \mathbf{P}_{0 \mid 0}$ and $\mathbf{R}_{k}$, tailored to the simulation and real signal selected scenarios. In future works, we are considering the use of adaptive Kalman filters to avoid tuning the EKF for a specific environment. More SA measurements campaigns are planned, particularly in scenarios with severe multipath.

\section{REFERENCES}

[1] M. G. Amin, P. Closas, A. Broumandan, and J. L. Volakis, "Vulnerabilities, threats, and authentication in satellite-based navigation systems [scanning the issue]," Proceedings of the IEEE, vol. 104, no. 6, pp. 1169-1173, June 2016.

[2] C. Fernandez-Prades, J. Arribas, and P. Closas, "Robust GNSS Receivers by Array Signal Processing: Theory and Implementation," Proceedings of the IEEE, vol. 104, no. 6, pp. 1207 - 1220, 2016.

[3] M. Skolnik, Introduction to Radar Systems, Tata McGraw Hill, 2003.

[4] S. Daneshmand, A. Broumandan, N. Sokhandan, and G. Lachapelle, "GNSS Multipath Mitigation with a Moving Antenna Array," IEEE Transactions on Aerospace and Electronic Systems, vol. 49, no. 1, pp. 693-698, jan 2013.

[5] Mohammad Hatef Keshvadi, Ali Broumandan, and Gerard Lachapelle, "Analysis of GNSS beamforming and angle of arrival estimation in multipath environments," in Institute of Navigation - International Technical Meeting 2011, ITM 2011, San Diego, CA, United states, 2011, pp. 427-435.

[6] T. Pany, N. Falk, B. Riedl, C. Stöber, J. Winkel, and H.-P. Ranner, "GNSS Synthetic Aperture Processing with Artificial Antenna Motion," in Proceedings of the 26th International Technical Meeting of The Satellite Division of the Institute of Navigation (ION GNSS+ 2013), sep 2013, pp. 3163-3171.

[7] Tao Lin, Ali Broumandan, John Nielsen, Cillian O Driscoll, and Gerard Lachapelle, "Robust Beamforming for GNSS Synthetic Antenna Arrays," in Proceedings of the 22nd International Technical Meeting of The Satellite Division of the Institute of Navigation (ION GNSS 2009), Savanha, GA, US, 2009, number September, pp. 387-401.

[8] Ali Broumandan, T Lin, and A Moghaddam, "Direction of arrival estimation of GNSS signals based on synthetic antenna array," International Technical Meeting of the Satellite Division of The Institute of Navigation, ION GNSS, , no. September, pp. 728-738, 2007.

[9] Avram K. Tetewsky and Frank E. Mullen, “Carrier Phase Wrap-Up Induced by Rotating GPS Antennas,” 1996.

[10] Elliott D. Kaplan and Christopher J. Hegarty, Understanding GPS: Principles and Applications, Artech House, 2nd edition, 2006.

[11] Paul D. Groves, "Principles of GNSS, inertial, and multisensor integrated navigation systems, 2nd edition [Book review]," IEEE Aerospace and Electronic Systems Magazine, vol. 30, no. 2, pp. 26-27, 2015.

[12] H L Van Trees, Detection, Estimation, and Modulation Theory, Optimum Array Processing, Detection, Estimation, and Modulation Theory. Wiley, 2002.

[13] Hatef Keshvadi, Ali Broumandan, and Garard Lachapelle, "Spatial characterization of GNSS multipath channels," International Journal of Antennas and Propagation, vol. 2012, 2012. 
[14] André Pousinho, Manuel Toledo, Teresa Ferreira, José A López-salcedo, Sergi Locubiche-serra, Gonzalo Seco-granados, Miguel Angel Ribot, Aleksandar Jovanovic, Cyril Botteron, and P Farine, "Adaptive Tracking Techniques in Non- Stationary Environments," in 28th International Technical Meeting of the Satellite Division of the Institute of Navigation, ION GNSS 2015, 2014, vol. 4.

[15] Melania Susi, Marcus Andreotti, Marcio Aquino, and Alan Dodson, "Tuning a Kalman filter carrier tracking algorithm in the presence of ionospheric scintillation," 2017.

[16] Jose A. Lopez-Salcedo, Jose A Del Peral-Rosado, and Gonzalo Seco-Granados, "Survey on robust carrier tracking techniques," 2014.

[17] Jordi Vila-Valls, Pau Closas, and Carles Fernandez-Prades, "Advanced KF-based methods for GNSS carrier tracking and ionospheric scintillation mitigation," in 2015 IEEE Aerospace Conference. mar 2015, pp. 1-10, IEEE.

[18] T. Pany and B. Eissfeller, "Demonstration of a Synthetic Phased Array Antenna for Carrier/Code Multipath Mitigation," in Proceedings of the 21st International Technical Meeting of the Satellite Division of The Institute of Navigation (ION GNSS 2008), sep 2008, pp. 663-668.

[19] Thomas Pany, Nico Falk, Bernhard Riedl, Carsten Stöber, Jón O. Winkel, and Franz-Josef Schimpl, "Innovation: Under Cover: Synthetic-Aperture GNSS Signal Processing," GPS World, vol. September, 2013.

[20] NSL, NSL Stereo: dual GNSS front-end datasheet, Nottingham Scientific Ltd (NSL), Nottingham, UK.

[21] Kai Borre, A Software-Defined GPS and Galileo Receiver, 2007.

[22] Spirent, Spirent GSS8000 GNSS Simulator datasheet.

[23] ANTCOM, Ed., Antcom GNSS Antennas catalogue-datasheet, ANTCOM Corporation, Torrance, CA, US, 2017. 